\title{
Gender differences in health anxiety and musculoskeletal symptoms during the COVID-19 pandemic
}

\author{
Muge Kirmizi $^{\mathrm{a}, \mathrm{b}, *}$, Gamze Yalcinkaya ${ }^{\mathrm{b}}$ and Yesim Salik Sengul ${ }^{\mathrm{c}}$ \\ ${ }^{a}$ Department of Physiotherapy and Rehabilitation, Faculty of Health Sciences, Izmir Katip Celebi University, Cigli, \\ Izmir, Turkey \\ ${ }^{\mathrm{b}}$ Institute of Health Sciences, Dokuz Eylul University, Balcova, Izmir, Turkey \\ ' School of Physical Therapy and Rehabilitation, Dokuz Eylul University, Balcova, Izmir, Turkey
}

Received 9 October 2020

Accepted 21 December 2020

\begin{abstract}
.
BACKGROUND: Health anxiety is often associated with musculoskeletal symptoms and gender, but there are limited studies that investigate these relationships during the COVID-19 pandemic.

OBJECTIVE: We aimed to compare the genders regarding health anxiety and musculoskeletal symptoms during the COVID-19 pandemic and to investigate the relationship of musculoskeletal symptoms with physical activity and health anxiety.

METHODS: Assessments were performed through an online questionnaire. Eighty-five males and eighty-five females were included by matching genders in terms of age, body mass index, education level, number of days spent at home during the pandemic, and physical activity. The Short Health Anxiety Inventory, the International Physical Activity Questionnaire-Short Form, and the Nordic Musculoskeletal Questionnaire were used to assess health anxiety, physical activity, and musculoskeletal symptoms, respectively.

RESULTS: Females had a higher level of health anxiety and more musculoskeletal symptoms than males $(p<0.05)$. There were weak to moderate correlations in females and weak correlations in males between musculoskeletal symptoms and health anxiety $(p<0.05)$. Also, a weak negative correlation was found between physical activity and musculoskeletal symptoms in both genders $(p<0.05)$.

CONCLUSIONS: Females are more anxious and have more musculoskeletal symptoms during the pandemic than males. Also, health anxiety is related to musculoskeletal symptoms in both genders.
\end{abstract}

Keywords: Pandemic, COVID-19, health anxiety, musculoskeletal pain, musculoskeletal symptoms

\section{Introduction}

The Coronavirus Disease 2019 (COVID-19) caused by a novel Coronavirus type (SARS-CoV-2) has threatened the health and lives of millions of people around

\footnotetext{
${ }^{*}$ Corresponding author: Muge Kirmizi, Department of Physiotherapy and Rehabilitation, Faculty of Health Sciences, Izmir Katip Celebi University, TR-35620, Cigli, Izmir, Turkey. Tel.: +90 2323293535; Fax: +90 2323860888; E-mail: mugekirmizi1@ gmail. com.
}

the world since it began in the Chinese city of Wuhan at the end of 2019 [1,2]. COVID-19 can lead to a wide variety of symptoms ranging from mild to severe and can progress to severe disease with critical respiratory symptoms and signs especially in elderly patients and those with chronic illness [3-5]. The World Health Organization has declared the COVID-19 outbreak a global pandemic on 11 March 2020 [6]. The first case in Turkey was confirmed on 11 March 2020. As of this writing, there were 323,000 confirmed cases and 8,384 confirmed deaths in Turkey [7]. 
The outbreak of COVID-19 has affected people's mental and physical health $[8,9]$. Health anxiety means worrying and experiencing anxiety due to a perceived threat to health and is one of the most predominant psychological symptoms during the pandemic [10-13]. Health anxiety exists along a continuum from a lack of health awareness to severe health anxiety [10]. Since health anxiety, which was reported to be associated with disability and loss of quality of life, has been seen more frequently during the pandemic, there is an increasing focus on the assessment and management of health anxiety in the current literature [11-15]. In Turkey, as in most other countries, studies show that anxiety has increased during the COVID-19 pandemic. Furthermore, many studies have been perfomed to determine the health anxiety-related factors during the pandemic, and the results have suggested that gender stands out as the predominant risk factor for health anxiety [16,17].

Musculoskeletal symptoms are presumed to increase during the COVID-19 pandemic due to the fact that people have lost their normal daily activity patterns through the lockdown and have become less physically active $[18,19]$. Moretti et al. have suggested that working from home during the COVID-19 pandemic causes worsening of musculoskeletal problems, particularly affecting the spine [20]. However, the relationship between the amount of physical activity and musculoskeletal symptoms during the pandemic is unknown. On the other hand, no study has yet investigated the relationship between health anxiety and musculoskeletal symptoms. It has been suggested that people with anxiety have a higher prevalence of musculoskeletal pain and vice-versa; and furthermore, anxiety reduces the pain threshold [21-24]. Also, psychosocial factors including anxiety have been typically recommended to be addressed in the management of musculoskeletal pain $[22,23,25]$. Given this link between anxiety and musculoskeletal pain, we assumed that investigating the relationship of health anxiety with musculoskeletal symptoms during the COVID-19 pandemic will provide valuable data. Also, we aimed to investigate this relationship separately for both genders because it has been suggested that there may be differences in the association between anxiety symptoms and related variables such as risk and protective factors, comorbidity, and influencing factors in females and males [26,27].

Overall, we have hypothesized that females would have a higher level of health anxiety during the last week of the pandemic. Also, musculoskeletal symptoms would be related to the amount of physical activity and health anxiety. In light of this information, the present study was designed to compare the genders regarding health anxiety and musculoskeletal symptoms during the COVID-19 pandemic and to investigate the relationship of musculoskeletal symptoms with the amount of physical activity and health anxiety.

\section{Materials and methods}

This gender comparison study was conducted following the ethical approval was obtained from Dokuz Eylul University Institutional Non-invasive Research Ethics Board (No: 5399-GOA, Protocol Number: 2020/11-36, Date: 01.06.2020). All procedures were conducted in accordance with the Declaration of Helsinki and all participants provided informed consent by clicking the consent button in the online form to participate in this study.

\subsection{Participants}

The total sample size was estimated based on the data from a previous report [28]. We reached 494 volunteers (320 females, 174 males). Of the 494 volunteers, 357 participants (257 females, 100 males) completed all questionnaires and answered all questions. Of the 100 males, 85 met all inclusion and exclusion criteria and were included in the statistical analyses. Of the $257 \mathrm{fe}$ males, 220 met all inclusion and exclusion criteria. We used MS Excel 2020 to form the female group and we selected 85 females by matching them with 85 males in terms of age, body mass index, education level, number of days spent at home during the pandemic, and the total amount of physical activity. The inclusion criteria were as follows: (1) between 18 and 60 years of age, (2) not working in an isolation ward for suspected or confirmed COVID-19, (3) living in an urban area, (4) not being tested positive for COVID-19, (5) not having a positive Polymerase Chain Reaction (PCR) test result, and (6) not having common symptoms for COVID-19 such as fever, cough, shortness of breath. The exclusion criteria were as follows: (1) leaving at least one of the questionnaires blank, (2) giving a missing answer in any of the questionnaires, (3) being diagnosed with a cardiopulmonary, neurological, metabolic, or psychiatric disorder, (4) being obese, or (5) being pregnant.

\subsection{Procedures}

Assessments were performed between 1 and 10 June 2020 through an online questionnaire created using 
Google Forms software. People were contacted through social media (Instagram, Facebook and Whatsapp) and then asked to participate in the study on a voluntary basis. The online survey was shared with the people who agreed to participate and they were asked to fill out all questionnaires. The online survey started with the informed consent form and also included questions about sociodemographic characteristics (age, gender, weight, height, education level), medical history, and the number of days spent at home during the pandemic, as well as the Short Health Anxiety Inventory, the Nordic Musculoskeletal Questionnaire, and the International Physical Activity Questionnaire-Short Form.

\subsubsection{The Short Health Anxiety Inventory}

The Short Health Anxiety Inventory (SHAI) is an 18-item measure that assesses the severity of health anxiety. Each item consists of four statements rating from 1 to 4 and the total score ranges between 0 and 54. The Turkish version of the SHAI has appropriate properties including good construct validity, internal consistency, and good test-retest reliability [29].

\subsubsection{The Nordic Musculoskeletal Questionnaire}

The Nordic Musculoskeletal Questionnaire (NMQ) was used to determine the musculoskeletal symptom loads. The NMQ contains 27 items covering nine different parts of the body (neck, shoulders, elbows, wrists/hands, upper back, lower back, hips/thighs, knees, ankles/feet) to investigate the existence of musculoskeletal symptoms in the last 12 months and last seven days. The Turkish version of the NMQ has good test-retest reliability, internal consistency, and construct validity [30]. We determined the overall physical loads of proximal and distal segments via the NMQ according to the formula by Hollman et al. [31]. The items of the NMQ are classified as follows: $0=$ neither symptoms nor restrictions, $1=$ symptoms in the last 12 months but not in the last 7 days and no restrictions, $2=$ symptoms in the last 12 months and either symptoms in the last 7 days or restrictions, and $3=$ symptoms in both the last 12 months and the last seven days and restrictions. The average of the score obtained from the upper and lower back, neck, shoulders, and hips/thighs was defined as the proximal musculoskeletal symptom load. Then, the distal musculoskeletal symptom load was determined by the average score of elbows, wrists/hands, knees, and ankles/feet.

\subsubsection{The International Physical Activity Questionnaire-Short Form}

The total amount of physical activity in the last seven days was assessed with the International Physical Activity Questionnaire-Short Form (IPAQ-SF). The questionnaire includes seven items, which provide information about the time spent for sitting, walking, medium and high level of physical activities. The total amount of physical activity was obtained by summing the scores of mentioned activities in calculating "MET-minutes per week". 8 MET, 4 MET and 3.3 MET values were used for high level of physical activity, medium level of physical activity, and walking, respectively. The Turkish version of IPAQ-SF was found to be valid and reliable [32].

\subsection{Statistical analyses}

The statistical analysis was performed on 170 participants ( 85 females, 85 males) using SPSS Statistics version 25 (IBM Corp., Armonk, NY, USA). The Kolmogorov-Smirnov test and histograms were used to determine whether data has a normal distribution. Weight, height, and body mass index showed normal distribution while age, the number of days spent at home during the pandemic, health anxiety, pysical activity, and musculoskeletal symptom loads did not show normal distribution. Descriptive statistics were presented with mean and standard deviation for normally distributed data also with median and quartiles for nonnormally distributed data. The Independent Samples $t$-test and Mann-Whitney U test were used to compare groups for normally and non-normally distributed data, respectively. Spearmen correlation coefficients were calculated separately for both genders to determine the correlation between physical activity, health anxiety, and musculoskeletal symptom loads. The $P$-value was set to 0.05 . The statistically significant correlation coefficients were interpreted according to the guide by Schober [33]. A correlation coefficient less than 0.10 indicates a negligible correlation, coefficients between 0.10 and 0.39 indicate weak correlation, coefficients between 0.4 and 0.69 indicate moderate correlation, coefficients between 0.7 and 0.89 indicate high correlation, and coefficients between 0.9 and 1 indicate very strong correlation.

\section{Results}

The final sample included 170 participants ( 85 females, 85 males). The sociodemographic characteristics of both groups are presented in Table 1. The female and male groups were matched according to age, body mass 
Table 1

Sociodemographic characteristics of the two groups

\begin{tabular}{|c|c|c|c|}
\hline & Females $(n=85)$ & Males $(n=85)$ & $p$ \\
\hline $\operatorname{Age}(\mathrm{y})_{[\text {median }(\mathrm{O} 1 / \mathrm{O} 3)]}^{\mathrm{a}}$ & $28(23-28)$ & $29(24-39)$ & 0.959 \\
\hline Weight $(\mathrm{kg})_{[\text {mean }(\mathrm{SD})]}^{\mathrm{b}}$ & $64.16(8.01)$ & $77.32(10.22)$ & $<0.001^{*}$ \\
\hline Height $(\mathrm{cm})_{[\operatorname{mean}(\mathrm{SD})]}^{\mathrm{b}}$ & $163.81(6.23)$ & $178.08(7.26)$ & $<0.001^{*}$ \\
\hline $\operatorname{BMI}\left(\mathrm{kg} / \mathrm{m}^{2}\right)_{[\operatorname{mean}(\mathrm{SD})]}^{\mathrm{b}}$ & $23.86(2.24)$ & $24.37(2.84)$ & 0.196 \\
\hline Number of days spent at home during the pandemic $\left.c_{[m e d i a n}^{\mathrm{a}}(\mathrm{O} 1 / \mathrm{Q} 3)\right]$ & $60(42.50-65)$ & $60(40-60.50)$ & 0.413 \\
\hline Total amount of physical activity (MET-min/week) ${ }_{[\text {median (Q1/Q3)] }}^{\mathrm{a}}$ & $792(387-1386)$ & $852(396-1386)$ & 0.419 \\
\hline \multicolumn{4}{|l|}{ Education level $_{[\mathrm{n}(\%)]}^{\mathrm{c}}$} \\
\hline Primary school & - & - & \\
\hline Secondary school & - & - & 0.444 \\
\hline High school & $7(8.20)$ & $9(10.60)$ & \\
\hline Bachelor & $78(91.80)$ & $76(89.40)$ & \\
\hline
\end{tabular}

Q1: The first quartile; Q3: The third quartile; SD: Standard deviation; BMI: Body mass index; ${ }^{\text {a }: ~ M a n n-W h i t n e y ~ U ~ t e s t ; ~}{ }^{b}$ : Independent samples $t$ test; ${ }^{c}$ : Pearson Chi-square test; ${ }^{*}: p<0.05$.

Table 2

Comparison of groups regarding health anxiety and musculoskeletal symptom loads

\begin{tabular}{|c|c|c|c|}
\hline Variables & $\begin{array}{c}\text { Females }(n=85) \\
\text { Median }(\mathrm{Q} 1 / \mathrm{Q} 3)\end{array}$ & $\begin{array}{l}\text { Males }(n=85) \\
\text { Median }(\mathrm{Q} 1 / \mathrm{Q} 3)\end{array}$ & $p^{\mathrm{a}}$ \\
\hline SHAI & $16(13-21)$ & $13(9-15)$ & $<0.001^{*}$ \\
\hline Proximal musculoskeletal symptom load & $1(0.60-1)$ & $0.60(0.30-0.90)$ & $<0.001^{*}$ \\
\hline Distal musculoskeletal symptom load & $0.25(0-1)$ & $0.25(0-0.50)$ & $0.014^{*}$ \\
\hline \multicolumn{4}{|c|}{ Musculoskeletal symptom loads of each region } \\
\hline Neck & $1(1-2)$ & $1(1-2)$ & $0.024 *$ \\
\hline Shoulders & $1(1-2)$ & $0(0-1)$ & $<\mathbf{0 . 0 0 1}^{*}$ \\
\hline Elbows & 0 & 0 & 0.325 \\
\hline Wrists/hands & 0 & 0 & 0.233 \\
\hline Upper back & $1(0-2)$ & $0(0-2)$ & $0.024 *$ \\
\hline Lower back & $1(1-3)$ & $1(0-1)$ & $<\mathbf{0 . 0 0 1}^{*}$ \\
\hline Hip/thighs & $0(0-1)$ & 0 & 0.108 \\
\hline Knees & $0(0-1)$ & $0(0-1)$ & $0.047^{*}$ \\
\hline Ankles/feet & $0(0-1)$ & 0 & 0.031* \\
\hline
\end{tabular}

Q1: The first quartile; Q3: The third quartile; SHAI, Short Health Anxiety Inventory; ${ }^{\text {a }}$ : Mann-Whitney U test; ${ }^{*}: p<0.05$.

index, education level, number of days spent at home during the pandemic, and total amount of physical activity when the groups were formed. Therefore, these variables did not significantly differ between the groups $(p>0.05)$.

The comparative statistics for health anxiety and musculoskeletal load scores among the gender groups are presented in Table 2 . Health anxiety and proximal and distal musculoskeletal symptom loads were significantly higher in females than males $(p<0.05)$. Musculoskeletal symptom loads in the neck, shoulders, upper and lower back, knees, and ankles/feet were also higher in females than males $(p<0.05)$.

Health anxiety was moderately correlated with proximal musculoskeletal symptom load and musculokeletal symptom load in the lower back in females (all $p<0.05 ;$ rho $=0.404$ and rho $=0.519$, respectively) . Health anxiety was also weakly correlated with distal musculoskeletal symptom load and musculokeletal symptom loads in the neck and knees in both groups (all $p<0.05$; for females rho $=0.307$, rho $=0.226$, and rho $=0.302$, respectively; for males rho $=0.265$, rho $=0.214$, and rho $=0.239$, respectively). Furthermore, there was a weak correlation between health anxiety and musculoskeletal symptom loads in elbows and hip/thighs in females (all $p<0.05$; rho $=0.296$ and rho $=0.344$, respectively) as well as between health anxiety, and proximal musculoskeletal symptom load and musculoskeletal symptom load in the upper back in males (all $p<0.05$; rho $=0.332$ and rho $=0.329$, respectively) (Table 3 ).

The total amount of physical activity had a negative and weak correlation with health anxiety in females (rho $=-0.221, p<0.05$ ), while those two variables did not significantly correlate in males $(p>0.05)$. Furthermore, the total physical activity was found to be weakly correlated with proximal musculoskeletal symptom load (rho $=-0.312, p<0.05)$ and musculokeletal 
Table 3

Correlation between total physical activity, health anxiety, and musculoskeletal symptoms in females and males

\begin{tabular}{|c|c|c|c|c|c|c|c|c|}
\hline & \multicolumn{4}{|c|}{ Females } & \multicolumn{4}{|c|}{ Males } \\
\hline & \multicolumn{2}{|c|}{ TPA } & \multicolumn{2}{|c|}{ SHAI } & \multicolumn{2}{|c|}{ TPA } & \multicolumn{2}{|c|}{ SHAI } \\
\hline & rho & $p$ & rho & $p$ & rho & $p$ & rho & $p$ \\
\hline TPA & 1 & - & -0.221 & 0.042* & 1 & - & 0.095 & 0.387 \\
\hline SHAI & -0.221 & $0.042 *$ & 1 & - & 0.095 & 0.387 & 1 & - \\
\hline PMSL & -0.312 & $0.004^{*}$ & 0.404 & $<\mathbf{0 . 0 0 1}^{*}$ & -0.149 & 0.174 & 0.332 & $0.002 *$ \\
\hline DMSL & -0.131 & 0.234 & 0.307 & $0.004^{*}$ & -0.093 & 0.395 & 0.265 & $0.014^{*}$ \\
\hline Neck & -0.262 & $0.015^{*}$ & 0.226 & $0.038^{*}$ & -0.241 & $0.027^{*}$ & 0.214 & 0.049* \\
\hline Shoulder & -0.096 & 0.382 & 0.175 & 0.109 & 0.070 & 0.522 & 0.212 & 0.051 \\
\hline Elbows & -0.015 & 0.892 & 0.296 & $0.006 *$ & -0.199 & 0.068 & 0.211 & 0.053 \\
\hline Wrists/hands & -0.219 & $0.044^{*}$ & 0.212 & 0.052 & -0.115 & 0.294 & 0.204 & 0.061 \\
\hline Upper back & -0.306 & $0.004^{*}$ & 0.166 & 0.130 & -0.067 & 0.540 & 0.329 & $0.002 *$ \\
\hline Lower back & -0.209 & 0.055 & 0.519 & $<\mathbf{0 . 0 0 1}^{*}$ & -0.197 & 0.071 & 0.192 & 0.078 \\
\hline Hip/thighs & -0.165 & 0.131 & 0.344 & 0.001* & -0.084 & 0.447 & 0.120 & 0.272 \\
\hline Knees & -0.086 & 0.436 & 0.302 & $0.005^{*}$ & 0.006 & 0.953 & 0.239 & $0.027^{*}$ \\
\hline Ankles/feet & -0.059 & 0.594 & 0.040 & 0.713 & 0.034 & 0.755 & 0.095 & 0.385 \\
\hline
\end{tabular}

TPA: The total amount of physical activity; SHAI: Short Health Anxiety Inventory; PMSL: Proximal musculoskeletal symptom load; DMSL: Distal musculoskeletal symptom load; rho: Spearman's rank correlation coefficient; ${ }^{*}: p<0.05$.

symptom load in the neck (rho $=-0.262, p<0.05)$, wrists/hands (rho $=-0.219, p<0.05$ ), and upper back (rho $=-0.306, p=0.004$ ) in females. However, the total amount of physical activity was significantly correlated only with musculokeletal symptom load in the neck in males (rho $=-0.241, p<0.05)$ (Table 3 ).

\section{Discussion}

The present study aimed to compare the genders regarding health anxiety and musculoskeletal symptoms during the COVID-19 pandemic and to investigate the relationship of musculoskeletal symptoms with the amount of physical activity and health anxiety. Our results suggested that females have a higher level of health anxiety and more musculoskeletal symptoms than males. Also, there were weak to moderate correlations in the female group and weak correlations in the male group between musculoskeletal symptoms and health anxiety. Furthermore, a weak negative correlation was found between physical activity and musculoskeletal symptoms in females and males.

Health anxiety is one of the most predominant psychological symptoms during the pandemic [10-13]. Similar to before the pandemic, health anxiety was reported to be more prevalent in females than males during the COVID-19 pandemic and the female gender has been identified as a potent predictor of increased health anxiety $[9,34]$. Similar to the findings of studies conducted in other countries, it has been reported that females are more psychologically affected by the
COVID-19 pandemic than men and the risk of anxiety in females is 2.1 times higher than in males in Turkish society $[16,17]$. In accordance with the abovementioned studies, our results also showed that females have a higher level of health anxiety during the COVID19 pandemic. Furthermore, we revealed that there are weak to moderate correlations between musculoskeletal symptom loads and health anxiety. People with anxiety have been reported to have a higher prevalence of musculoskeletal pain and vice-versa [22,24]. Furthermore, it has been suggested that anxiety reduces the pain threshold and pain can be a common symptom or a good indicator of anxiety disorder [21,23]. Therefore, musculoskeletal symptoms can be taken into consideration in the management of health anxiety even during the pandemic and vice-versa.

Females have been frequently reported to have a lower pain threshold and pain tolerance compared to males $[35,36]$. Our results also showed that females have more musculoskeletal symptoms than males. Considering the relationship between musculoskeletal symptoms and health anxiety, the fact that females experienced more musculoskeletal symptoms than males may be one of the causes of a higher level of health anxiety in females. Furthermore, we also found that physical activity is weakly correlated with anxiety in females. Sfendla et al. reported that people who are more physically active have a lower level of anxiety than people who are insufficiently active during the COVID-19 period [37]. Thus, the amount of physical activity can be accepted as one of the factors related to health anxiety during the pandemic. 
There is insufficient studies investigating how lockdown affects musculoskeletal symptoms, although musculoskeletal symptoms are presumed to increase during the COVID-19 pandemic because people have lost their normal daily activity patterns through the lockdown and have become less physically active $[18,19]$. Fallon et al. reported that the COVID-19 lockdown causes an increase in pain levels in people with chronic pain. They also identified pain catastrophizing and self-perceived changes in physical activity as important predictors of self-perceived changes in pain [38]. Furthermore, Celenay et al. found that people who stayed at home during the COVID-19 pandemic have more musculoskeletal complaints than those who continued to work, suggesting that it may be due to the alterations of daily life activities with increased time spent at home and physical inactivity. Also, physical inactivity has been reported to cause musculoskeletal complaints, especially spinal pain [39]. In accordance with the abovementioned studies, we found that there is a weak negative correlation between physical activity and musculoskeletal symptom loads in the regions of the spine. Musculoskeletal disorders have a multifactorial etiology and physical activity is just one of many factors [40]. Future studies examining the course and predictors of musculoskeletal symptoms in the pandemic may be useful in terms of the current and future public health and burden on the healthcare system.

There are several limitations in the present study. The main limitation is that we formed two groups and matched them in terms of age, body mass index, education level, number of days spent at home during the pandemic, and the amount of physical activity. Female gender, accompanying chronic disease, previous psychiatric history, and living with a high-risk individual at home were reported to be risk factors for health anxiety during the COVID-19 pandemic in Turkish society [17]. In this study we included people living in urban areas, who were not diagnosed with a cardiopulmonary, neurological, metabolic, or psychiatric disorder, and did not work in an isolation ward for suspected or confirmed COVID-19. However, we did not take into consideration living with a high-risk individual at home and did not question whether they have a physical anomaly or not. Furthermore, although we revealed that health anxiety is related to musculoskeletal symptoms during the COVID-19 pandemic, we did not clarify the course and predictors of musculoskeletal symptoms.

\section{Conclusions}

The present study showed that females have a higher level of health anxiety and more musculoskeletal symptoms during the COVID-19 pandemic than males, and health anxiety is related to musculoskeletal symptoms in both males and females. Considering that increased health anxiety threatens not only mental health but also physical health is widespread during the COVID-19 outbreak, thus addressing the assessment of musculoskeletal symptoms in the management of health anxiety and vice-versa may provide valuable data in both genders. Also, encouraging to be more physically active during the lockdown may be useful to improve musculoskeletal symptoms.

\section{Conflict of interest}

None to report.

\section{References}

[1] Sohrabi C, Alsafi Z, O’Neill N, Khan M, Kerwan A, Al-Jabir A, et al. World health organization declares global emergency: a review of the 2019 novel coronavirus (COVID-19). Int J Surg (Internet). 2020; 76(February): 71-6.

[2] Shereen MA, Khan S, Kazmi A, Bashir N, Siddique R. COVID-19 infection: origin, transmission, and characteristics of human coronaviruses. J Adv Res (Internet). 2020; 24: 91-8.

[3] Liu Y, Yan LM, Wan L, Xiang TX, Le A, Liu JM, et al. Viral dynamics in mild and severe cases of COVID-19. Lancet Infect Dis (Internet). 2020; 20(6): 656-7.

[4] Gandhi RT, Lynch JB, del Rio C. Mild or Moderate Covid-19. N Engl J Med. 2020; 1-9.

[5] Berlin DA, Gulick RM, Martinez FJ. Severe Covid-19. N Engl J Med. 2020; 1-10.

[6] Cucinotta D, Vanelli M. WHO declares COVID-19 a pandemic. Acta Biomed. 2020; 91(1): 157-60.

[7] Republic of Turkey Ministry of Health. Republic of Turkey Ministry of Health (Internet). Republic of Turkey Ministry of Health. 2020 (cited 2020 Oct 3). Available from: https://covid 19.saglik.gov.tr/.

[8] Salari N, Hosseinian-Far A, Jalali R, Vaisi-Raygani A, Rasoulpoor S, Mohammadi M, et al. Prevalence of stress, anxiety, depression among the general population during the COVID19 pandemic: a systematic review and meta-analysis. Global Health. 2020; 16(1): 1-11.

[9] Vindegaard N, Eriksen Benros M. COVID-19 pandemic and mental health consequences: systematic review of the current evidence. Brain Behav Immun (Internet). 2020; (May): 1-12.

[10] Vigo D, Patten S, Pajer K, Krausz M, Taylor S, Rush B, et al. Mental Health of Communities during the COVID-19 Pandemic. Can J Psychiatry (Internet). 2020; 1-7.

[11] Cannito L, Di A, University D, D'annunzio G. Health anxiety and attentional bias toward virus-related stimuli during the COVID-19 pandemic. Res Sq (Internet). 2020; 1-12. 
[12] Jungmann SM, Witthöft M. Health anxiety, cyberchondria, and coping in the current COVID-19 pandemic: Which factors are related to coronavirus anxiety? J Anxiety Disord. 2020; 73(May).

[13] Nicomedes CJC, Avila RMA. An analysis on the panic during COVID-19 pandemic through an online form. J Affect Disord. 2020; 276(June): 14-22.

[14] Bobevski I, Clarke DM, Meadows G. Health anxiety and its relationship to disability and service use: findings from a large epidemiological survey. Psychosom Med. 2016; 78(1): 13-25.

[15] Fink P, Ørnbøl E, Christensen KS. The outcome of Health anxiety in primary care. A two-year follow-up study on health care costs and self-rated health. PLoS One. 2010; 5(3).

[16] Çoşkun A, Erkasap A, Aksit M. Investigation results of the anxiety impact of COVID-19 pandemic on individuals. Int J Res Bus Soc Sci (2147-4478). 2020; 9(3): 80-4.

[17] Özdin S, Bayrak Özdin Ş. Levels and predictors of anxiety, depression and health anxiety during COVID-19 pandemic in Turkish society: the importance of gender. Int J Soc Psychiatry (Internet). 2020; 66(5): 504-11.

[18] Joseph SJ, Shoib S, Sg T, Bhandari SS. Psychological concerns and musculoskeletal pain amidst the COVID-19 lockdown. Open J Psychiatry Allied Sci (Internet). 2020; 11(2): 137-9.

[19] Memari A, Shariat A, Anastasio AT. Rising incidence of musculoskeletal discomfort in the wake of the COVID-19 crisis Work (Internet). 2020; 66(4): 751-3.

[20] Moretti A, Menna F, Aulicino M, Paoletta M, Liguori S, Iolascon G. Characterization of home working population during covid-19 emergency: a cross-sectional analysis. Int J Environ Res Public Health. 2020; 17(17): 1-13.

[21] Rhudy JL, Meagher MW. Fear and anxiety: divergent effects on human pain thresholds. Pain. 2000; 84(1): 65-75.

[22] Vlaeyen JWS, Linton SJ. Fear-avoidance and its consequences in chronic musculoskeletal pain: a state of the art. Pain. 2000; 85(3): 317-32.

[23] Means-Christensen AJ, Roy-Byrne PP, Sherbourne CD, Craske MG, Stein MB. Relationships among pain, anxiety, and depression in primary care. Depress Anxiety. 2008; 25(7): 593600.

[24] Lucchetti G, Oliveira AB, Mercante JPP, Peres MFP. Anxiety and fear-avoidance in musculoskeletal pain. Curr Pain Headache Rep. 2012; 16(5): 399-406.

[25] Bijker L, Sleijser-Koehorst MLS, Coppieters MW, Cuijpers P, Scholten-Peeters GGM. Preferred self-administered questionnaires to assess depression, anxiety and somatization in people with musculoskeletal pain - a modified delphi study. J Pain. 2020; 21(3-4): 409-17.

[26] Christiansen DM. Examining Sex and Gender Differences in Anxiety Disorders. In: A Fresh Look at Anxiety Disorders (Internet). InTech; 2015. p. 13.
[27] Riecher-Rössler A. Sex and gender differences in mental disorders. The Lancet Psychiatry. 2017; 4(1): 8-9.

[28] Jensen I, Nygren A, Gamberale F, Goldie I, Westerholm P. Coping with long-term musculoskeletal pain and its consequences: is gender a factor? Pain. 1994; 57(2): 167-72.

[29] Aydemir Ö, Kirpinar I, Sati T, Uykur B, Cengisiz C. Saglık anksiyetesi ölçegi'nin Türkçe için güvenilirlik ve geçerlilik çalışması. Noropsikiyatri Ars. 2013; 50(4): 325-31.

[30] Kahraman T, Genç A, Göz E. The nordic musculoskeletal questionnaire: cross-cultural adaptation into Turkish assessing its psychometric properties. Disabil Rehabil. 2016; 38(21): 2153-60.

[31] Hollmann S, Klimmer F, Schmidt KH, Kylian H. Validation of a questionnaire for assessing physical work load. Scand J Work Environ Heal. 1999; 25(2): 105-14.

[32] Saglam M, Arikan H, Savci S, Inal-Ince D, Bosnak-Guclu M, Karabulut E, et al. International physical activity questionnaire: reliability and validity of the Turkish version. Percept Mot Skills. 2010; 111(1): 278-84.

[33] Schober P, Schwarte LA. Correlation coefficients: appropriate use and interpretation. Anesth Analg. 2018; 126(5): 1763-8.

[34] Rettie H, Daniels J. Coping and Tolerance of Uncertainty: Predictors and Mediators of Mental Health During the COVID19 Pandemic. Am Psychol. 2020.

[35] Bartley EJ, Fillingim RB. Sex differences in pain: a brief review of clinical and experimental findings. Br J Anaesth. 2013; 111(1): 52-8.

[36] Fillingim RB, King CD, Ribeiro-Dasilva MC, Rahim-Williams B, Riley JL. Sex, gender, and pain: a review of recent clinical and experimental findings. J Pain (Internet). 2009 May; 10(5): 447-85.

[37] Sfendla A, Hadrya F. Factors associated with psychological distress and physical activity during the COVID-19 pandemic. Heal Secur (Internet). 2020; 18(6)

[38] Nicholas Fallon, Christopher Brown, Hannah Twiddy, Eleanor Brian, Bernhard Frank, Turo Nurmikko AS. Adverse effects of COVID-19 related lockdown on pain, physical activity and psychological wellbeing in people with chronic pain. medRxiv (Internet). 2020; Preprint.

[39] Toprak Celenay S, Karaaslan Y, Mete O, Ozer Kaya D. Coronaphobia, musculoskeletal pain, and sleep quality in stay-at home and continued-working persons during the 3-month Covid-19 pandemic lockdown in Turkey. Chronobiol Int (Internet). 2020; 00(00): 1-8

[40] Evanoff B, Dale AM, Descatha A. A conceptual model of musculoskeletal disorders for occupational health practitioners. Int J Occup Med Environ Health. 2014; 27(1): 145-8. 